\title{
Early experience of TIARA transcatheter mitral valve replacement system
}

\author{
Anson Cheung \\ Division of Cardiothoracic Surgery, St. Paul's Hospital, University of British Columbia, Vancouver, British Columbia, Canada \\ Correspondence to: Anson Cheung, MD. 1080 Burrard Street, Vancouver, BC V6Z 1Y6, Canada. Email: acheung@providencehealth.bc.ca.
}

\begin{abstract}
Mitral regurgitation (MR) is a common valvular disorder that can result in congestive heart failure and cardiovascular death. Patient suffering from this condition are frequently not referred and thus the condition is under-treated. Surgery remains the gold standard treatment for mitral valve disease, however, catheter based mitral valve interventions, including repair and replacement, are being employed to treat patients who are deemed high or prohibitive risk for conventional surgery. TIARA is one of the many newly developed transcatheter mitral valve replacement (TMVR) systems that may address this unmet need.
\end{abstract}

Keywords: Mitral valve; transcatheter; replacement

Submitted Aug 29, 2018. Accepted for publication Sep 14, 2018.

doi: 10.21037 /acs.2018.09.05

View this article at: http://dx.doi.org/10.21037/acs.2018.09.05

\section{TIARA transcatheter mitral valve replacement (TMVR) system}

The TIARA TMVR (Neovasc Inc., Richmond, BC, Canada) is a transapical access-based system that is designed to treat mitral regurgitation (MR). The TIARA valve prosthesis consists of a nitinol self-expanding frame that is saddle shaped to mimic the geometry of the native mitral annulus (Figure 1). Three bovine pericardial leaflets provide the coaptation function and a large atrial skirt seals the mitral inflow to prevent paravalvular leak (PVL). Device fixation is provided by radial expansion of the TIARA and axial anchorage is achieved with ventricular tabs. Two anterior tabs anchor the valve onto the fibrous aortomitral curtain and a single posterior tab lands behind the posterior mitral leaflet onto the muscular posterior shelf of the mitral annulus (1-3). At present, a 35 and $40 \mathrm{~mm}$ device are available to treat a wide range of annular dimensions. Sizing is based on careful assessment of the mitral annular dimensions by contrast-enhanced multi-slice computed tomography (CT). TIARA valves are delivered by a sheathless system via the left ventricle (LV), a 36-French (Fr) system for the $35 \mathrm{~mm}$ valve and a $40 \mathrm{Fr}$ system for the larger valve. An atraumatic and self-dilating tip allows easy entry into the LV. Valve delivery, deployment and resheathing is achieved by a simple turn knob mechanism.

\section{Patient screening}

Identifying suitable candidates for TMVR therapy has been a challenge for all devices. Exclusion for potential TMVR therapy can be clinical, anatomical and/or device-specific. Extensive investigations include coronary angiogram, transthoracic and transesophageal echocardiography (TEE) and cardiac CT is performed in all patients. Patients with significant co-morbidities, poor ventricular function with a LVEF $<20 \%$, severe right ventricle dysfunction and irreversible pulmonary hypertension are deemed unsuitable. Echocardiographic eligibility criteria include severe symptomatic MR (stage D) by 2014 AHA/ACC Valvular Heart Disease Guidelines classification, as determined by Core Laboratory.

Contrast-enhanced gated CT data acquisition of the entire cardiac cycle must be acquired to determine the dimensions of the mitral annulus, the presence of annular calcification, subvalvular complexes, the $L V$ and left ventricular outflow tracts (LVOT). The risk for potential LVOT obstruction can be determined with simulated device implants and the anticipated residual neo-LVOT crosssectional area can be calculated. Patients with a predicted 

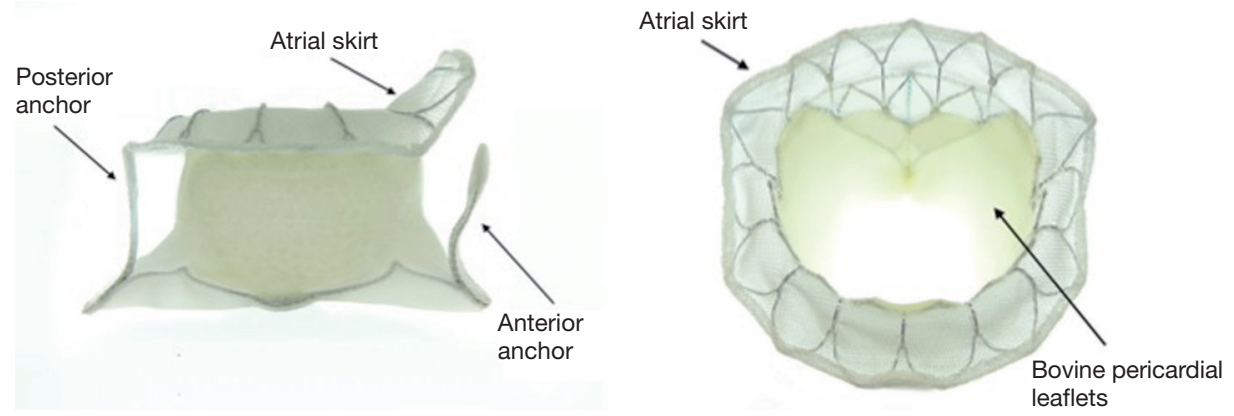

Figure 1 The Neovasc TIARA valve prosthesis.

Table 1 Sizing chart for the 35 and $40 \mathrm{~mm}$ TIARA valve
\begin{tabular}{llllll} 
Size $(\mathrm{mm})$ & Mitral annulus circumference $(\mathrm{cm})$ & Atrial height $(\mathrm{cm})$ & Annulus area $\left(\mathrm{cm}^{2}\right)$ & Distance \\
\cline { 4 - 6 } & $9.3-11.0$ & & & A-P $(\mathrm{mm})$ & C-C $(\mathrm{mm})$ \\
\hline 35 & $11.0-12.5$ & $\geq 5.0$ & $6.5-9.0$ & $26.5-30.0$ & $31.0-35.0$ \\
\hline 0 & & & $9.0-12.0$ & $30.0-34.0$ & $35.0-40.0$ \\
\hline
\end{tabular}

neo-LVOT area of $\geq 2.0 \mathrm{~cm}^{2}$ at end-systole were considered eligible for TIARA.

CT data including the entire rib cage was used to determine the optimal intercostal space for transapical access. Anatomical suitabilities are mainly assessed by echocardiography and cardiac CT. In addition to annular dimensions, cardiac CT provides invaluable information for determining other anatomical details, such as LVOT dimension, angle and anatomical fit of the TIARA device (4). Common patient exclusions include poor LV function, excessive mitral annular dimensions, absence of posterior shelf and high risk of LVOT obstruction. Table 1 illustrates the sizing criteria for the two current devices.

\section{Case example and implant procedure}

An 80-year-old man with ischemic cardiomyopathy, previous myocardial infarction, coronary artery bypass grafting, peripheral vascular disease with repair of abdominal aortic aneurysm and chronic renal insufficiency on good medical therapy. This patient suffered from NYHA class III heart failure with an ejection fraction of $25 \%$ and severe functional MR. His STS and EuroSCORE II risk score for mitral valve replacement (MVR) were $8.4 \%$ and $28.4 \%$ respectively. The heart team's assessment deemed that the patient was at excessive risk for open MVR and not a suitable candidate for transcatheter leaflet plication therapy secondary to a retracted and short posterior MV leaflet. However, he was an excellent candidate for a $40 \mathrm{~mm}$ TIARA TMVR after careful screening (Figure $2 A, B$ ). The implant procedure was performed in a hybrid operating room with guidance from fluoroscopy and TEE.

Close intra-procedural collaboration from all heart team members, with the presence of a cardiac surgeon, interventional cardiologist and echocardiographer, is crucial to the success of the procedure. The ideal location of the left anterior mini-thoracotomy over the $\mathrm{LV}$ puncture site is guided by pre-procedural CT imaging and surface ultrasound. Placement of two horizontal mattress pledgeted sutures provided hemostasis of the LV puncture site. Crossing of the MV is achieved with a soft $\mathrm{J}$ wire and is later exchanged for a 0.035-inch Amplatz Extra-Stiff wire (Boston Scientific Corp., Indianapolis, USA). Subvalvular entanglement was assessed and ruled out by TEE. The TIARA TMVR delivery system was inserted across the MV into the left atrium (LA), and subsequently, the atrial skirt of the TIARA system is delivered across the valve. Threedimensional TEE guidance is used to ensure anatomical alignment of D-shaped TIARA device to match the geometry of the MV annulus.

Once aligned, the atrial skirt was seated onto the atrial MV annulus by withdrawal of the system ventricularly. Device resheathing, repositioning and retrieval can be 

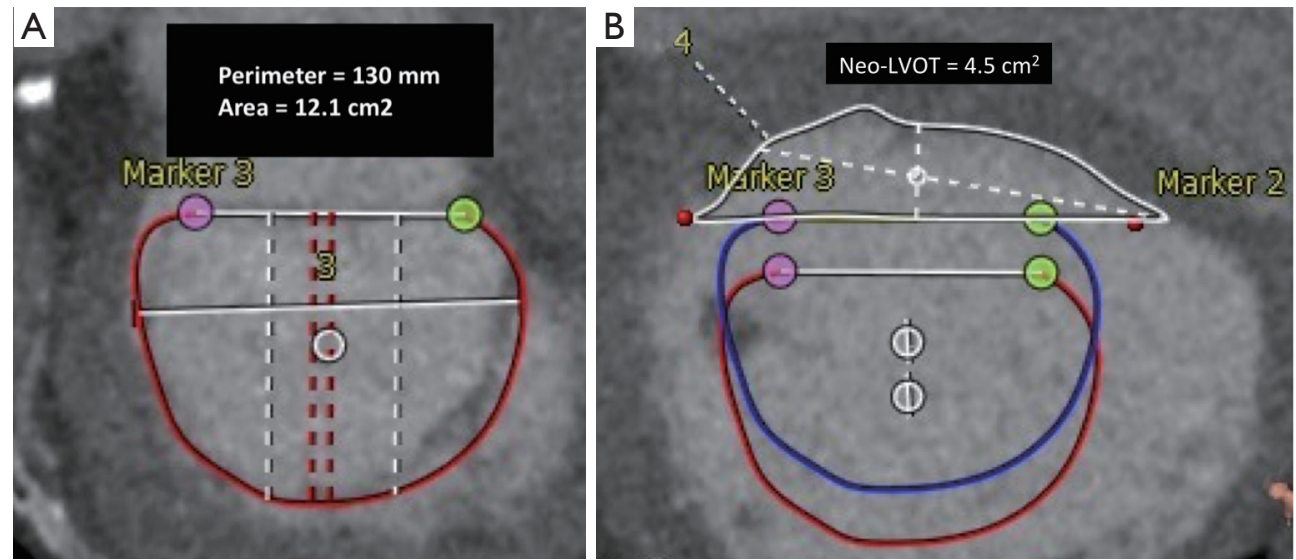

Figure 2 Pre-procedural CT assessment of the mitral apparatus. (A) CT assessment of suitability for TIARA implant with measurements of annular dimensions; (B) simulated TIARA implantation with calculated neo-LVOT area. CT, computed tomography; LVOT, left ventricular outflow tract.
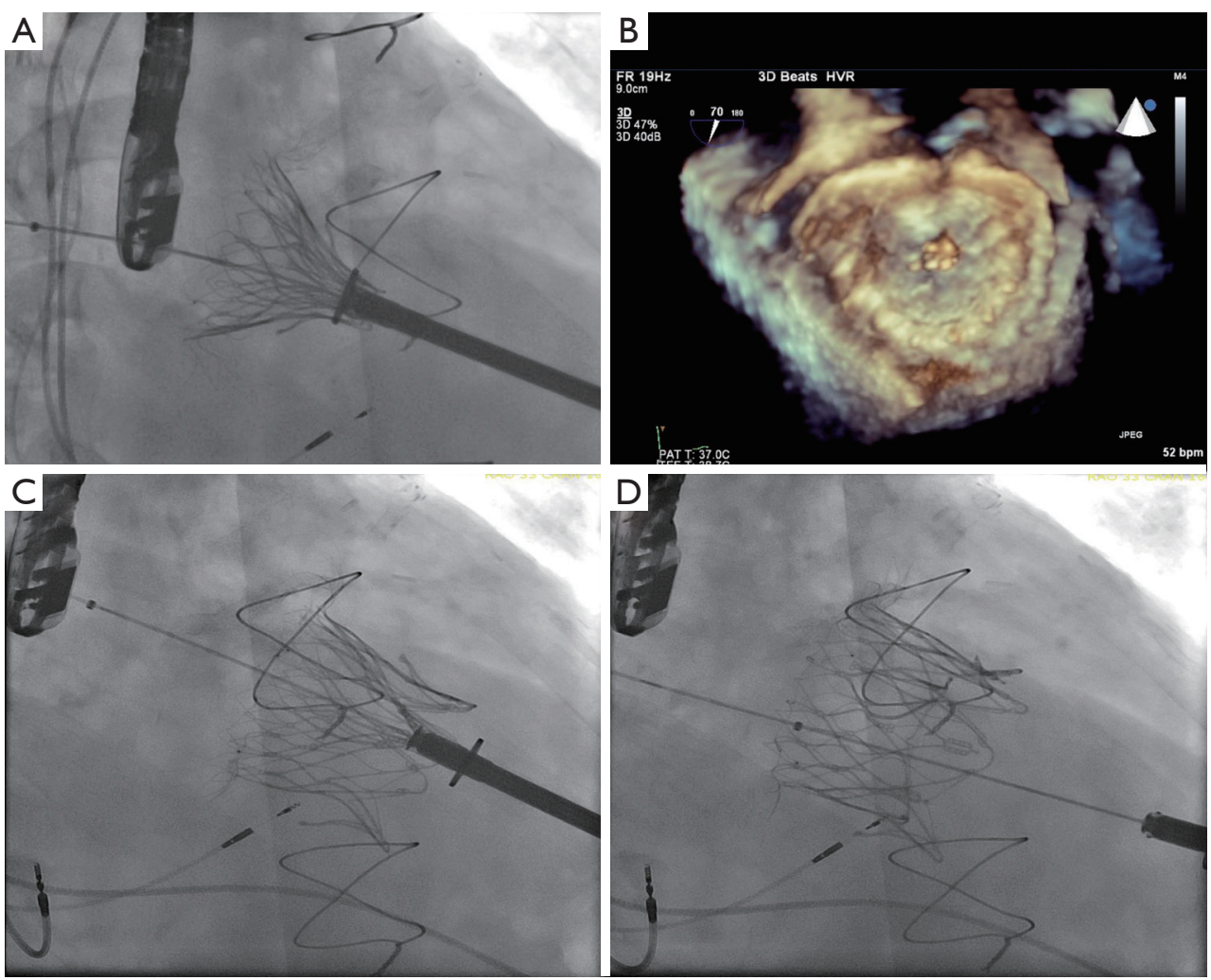

Figure 3 Procedural steps of a transapical TIARA implantation. (A) Unsheathing of atrial skirt; (B) TIARA rotation to align with the geometry of the native mitral annulus; (C) release of the two anterior anchoring tabs; (D) full deployment of the TIARA valve.

performed up to this stage of procedure. The ventricular tabs and the ventricular skirt were then released with further unsheathing (Figure $3 A, B, C, D$ ). Cardiopulmonary bypass support was not necessary and hemodynamic stability was maintained throughout the procedure. Excellent device seating was achieved with a mean transvalvular gradient of $2 \mathrm{mmHg}$, absence of LVOT obstruction and no PVL (Figure 4). The patient did not require blood product 


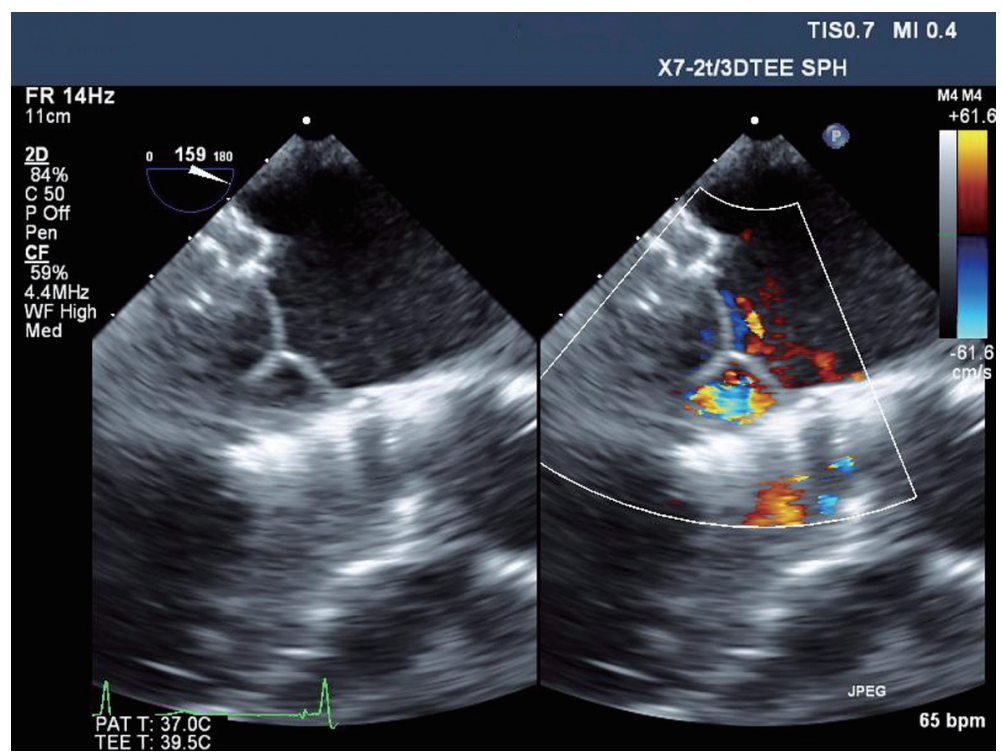

Figure 4 Post implant echocardiogram demonstrated a well seated TIARA valve with no mitral regurgitation or paravalvular leak.

transfusion, was extubated in the operating theatre and had an uneventful post-operative course.

\section{Clinical updates}

The first-in-human TIARA implantation was performed in 2014 by the team at St. Paul's Hospital, Vancouver, Canada (5). Many successful implantations have been conducted under compassionate access programs in Canada, Italy, Germany, Switzerland and Israel. The longest surviving patient from Canada has passed her 4-year anniversary with a well-seated, functioning prosthesis, with excellent hemodynamics and with minimal heart failure symptoms (NYHA class II). Currently, the TIARA TMVR programs include the TIARA-I Early Feasibility and the TIARA-II European CE Mark trials.

The TIARA-I is a multinational, multicenter trial being conducted in Canada, the US and Belgium, with the primary objectives being to assess the 30 -day safety outcomes in high-risk surgical patients suffering from severe MR. Secondary objectives include device performance, adverse events and clinical outcomes up to one year. The first enrollment and successful implantation was carried out in Belgium by Dr. Stefan Verhedye's team in 2014. The TIARA-I study is actively enrolling and anticipating full enrollment at the end of 2018. The TIARA-II CE Mark Trial is actively enrolling in Germany, Italy and the United Kingdom. Additional sites are being activated in Germany,
Italy and Israel. Thus far, over 50 patients have been treated with the TIARA TMVR with $95 \%$ implant success rates and no intraoperative mortality reported. The 30-day mortality in this very high-risk cohort was $8.5 \%$.

\section{Conclusions}

Many patients with severe MR are not currently offered treatment. Transcatheter mitral therapy provides additional options for patients that are deemed at too high a risk for surgical correction. The TIARA TMVR system is a TMVR technology specifically designed to address this pathology. The transventricular implant procedure is straight-forward, reproducible and safe. Correction of MR is complete with excellent hemodynamics. Ongoing implantations in the TIARA-I and II clinical trials will further provide important clinical data for TMVR in the treatment of mitral insufficiency.

\section{Acknowledgements}

None.

\section{Footnote}

Conflicts of Interest: A Cheung is Consultant and proctor, Neovasc Inc., Principle investigator, Tiara-1 Feasibility Trial. 


\section{References}

1. De Backer O, Piazza N, Banai S, et al. Percutaneous transcatheter mitral valve replacement: an overview of devices in preclinical and early clinical evaluation. Circ Cardiovasc Interv 2014;7:400-9.

2. Banai S, Jolicoeur EM, Schwartz M, et al. Tiara: A Novel Catheter-Based Mitral Valve Bioprosthesis: Initial Experiments and Short-Term Pre-Clinical Results. J Am Coll Cardiol 2012;60:1430-1.

Cite this article as: Cheung A. Early experience of TIARA transcatheter mitral valve replacement system. Ann Cardiothorac Surg 2018;7(6):787-791. doi: 10.21037/ acs.2018.09.05
3. Banai S, Verheye S, Cheung A, et al. Transapical mitral implantation of the Tiara bioprosthesis: pre-clinical results. JACC Cardiovasc Interv 2014;7:154-62.

4. Blanke P, Naoum C, Dvir D, et al. Predicting LVOT Obstruction in Transcatheter Mitral Valve Implantation: Concept of the Neo-LVOT. JACC Cardiovasc Imaging 2017;10:482-5.

5. Cheung A, Webb J, Verheye S, et al. Short-term results of transapical transcatheter mitral valve implantation for mitral regurgitation. J Am Coll Cardiol 2014;64:1814-9. 